\title{
Caffeine Supplementation Strategies Among Endurance Athletes
}

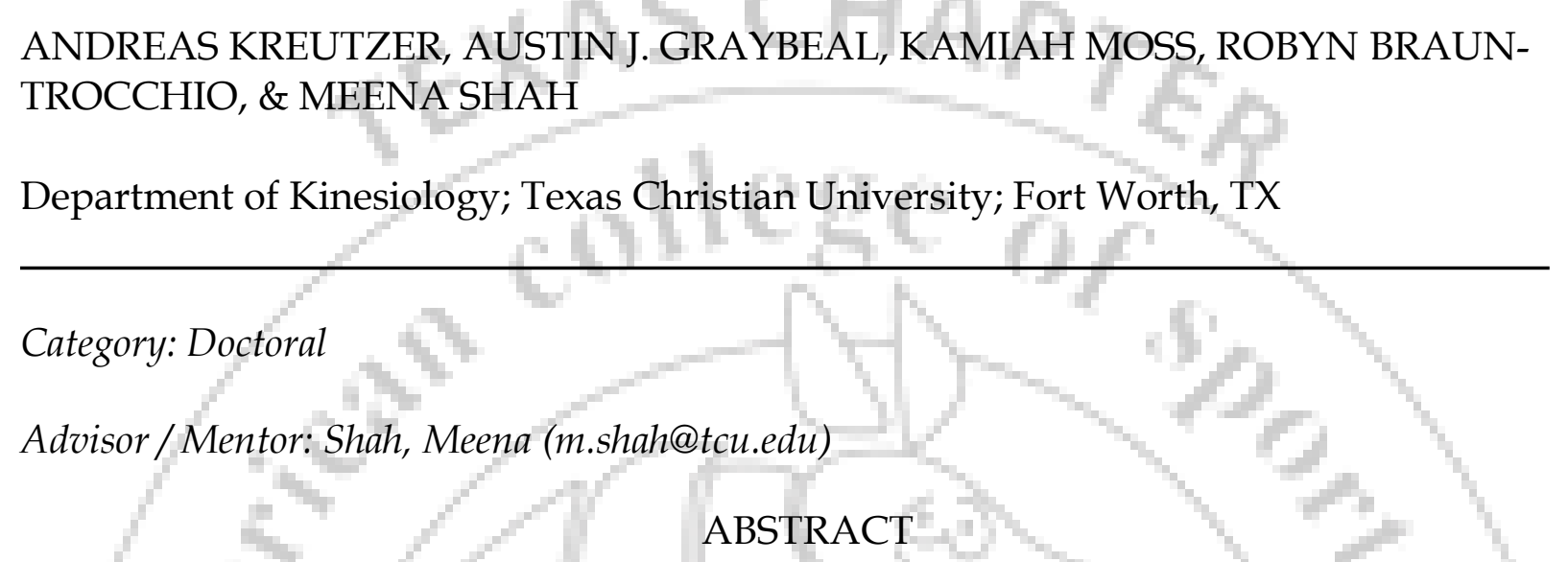

Caffeine is widely accepted as an ergogenic aid for endurance performance. Many laboratory studies use doses of 3-6 mg/ $\mathrm{kg}$ of caffeine $60 \mathrm{~min}$ prior to exercise. It is unclear if endurance athletes employ similar supplementation schemes in practice. Further, there is a paucity of data regarding caffeine consumption in this population. PURPOSE: The purpose of this study was to investigate caffeine supplementation strategies and consumption among endurance athletes. METHODS: A survey conducted on Qualtrics returned responses regarding caffeine supplementation from 247 endurance athletes $(\mathrm{f}=129, \mathrm{~m}=118$; age $=40.4 \pm 18.4 \mathrm{y} ;$ pro $=11$, current $/$ former collegiate athlete $=67$, recreational $=169$; running $=95$, triathlon $=$ 80 , cycling $=54$, other $=18$; training days per week $=5.4 \pm 1.3$ ). Descriptive statistics were calculated using SPSS V26. Pearson chi-square tests of independence were performed to investigate potential associations between a variety of grouping variables and caffeine use. Further, supplementation schemes were analyzed. Finally, athletes' perception of the effectiveness of caffeine were examined. RESULTS: The majority of participants reported habitual caffeine consumption $(84.2 \% ; 34.8 \%$ multiple times daily). Yet, only $23.5 \%$ reported using caffeine supplements. A greater percentage of men (30.5\%) used caffeine supplements compared with women $(17.1 \% ; p=.013)$. Athlete status was significantly associated with caffeine consumption $(p=.004)$. Caffeine use was more prevalent among professional $(36.4 \%)$ and recreational athletes $(28.4 \%)$ compared with current/former collegiate athletes $(9.0 \%)$. There were no significant differences in caffeine supplementation when comparing across type of sport $(p=.505)$, household income $(p=.191)$, education $(p=.453)$ or working with a coach $(p=.560)$. While not statistically significant $(p=.064), 53.4 \%$ of those using caffeine supplements reported placing among the top 3 in their age group in the past year, compared with only $39.7 \%$ of those not using caffeine supplements. Sixty-eight athletes $(27.5 \%)$ reported that they specifically timed caffeine supplementation around training $(60.3 \%$ only before, $14.7 \%$ only during, $25.0 \%$ before and during sessions). Seventy-seven (31.2\%) athletes reported timing caffeine intake around races (55.8\% before, $13.0 \%$ during, 31.2\% both). Of those reporting specific timing of caffeine use, $47.3 \%$ and $33.9 \%$ reported consuming caffeine within 30 min of training sessions and races respectively; $40.0 \%$ and $35.5 \%$ used caffeine $30-60 \mathrm{~min}$ before training and races; $12.7 \%$ and $36.6 \%$ reported taking caffeine $>60 \mathrm{~min}$ before training and races. The most frequently reported interval of supplementation during training $(64.0 \%)$ and races $(45.2 \%)$ was every $60-90$ minutes. Those reporting specific amounts of caffeine consumed before training $(n=27)$ and races $(n=14)$, used $1.8 \pm 1.0$ $\mathrm{mg} / \mathrm{kg}$ and $2.4 \pm 1.3 \mathrm{mg} / \mathrm{kg}$ respectively. On average, $53.6 \%$ and $39.1 \%$ of athletes reported that caffeine exerted no effects to only minor effects during various types of training and racing respectively. A greater percentage of athletes reported moderate and major effects during more intense training as well as longer training sessions and races (52.7 - 72.7\%). CONCLUSION: Most athletes in the present study did not follow typical laboratory protocols that have elicited ergogenic effects of caffeine. Better education among athletes and coaches or research into more diverse supplementation schemes are needed. 